\title{
Research on the Binder Distribution Depth and Evenness in Coated Paper
}

\author{
Ying $\mathrm{Li}^{1}$, a Jie Zhang ${ }^{1}$ and Haishan $\mathrm{Li}^{2}$ \\ ${ }^{1}$ Faculty of Mechanical and Electrical Engineering, Kunming University of Science and Technology, \\ Kunming, 650093, China \\ ${ }^{2}$ Kunming Richfun Printing Co., Ltd, Kunming, 650217, China \\ ${ }^{a}$ Email:haishanying@126.com
}

Keywords: Binder distribution; evenness; Z- directional distribution; Coated paper; LSCM

\begin{abstract}
In order to characterizing the binder distribution, Changed drying temperatures were used to alter binder distribution in coating drying process, which was used to investigate into the effect of drying condition on the binder distribution and characterize the binder migration in the method of numerical and visual analysis by Laser Scanning Confocal Microscope (LSCM). In this research, Rhodamine B was used to stain the binder and Rhodamine B was as the probe to characterize the z-directional distribution of the binder using LSCM. The results illustrated that it was a reliable and effective method to characterize the z- directional distribution of binder by LSCM. The result showed that the high drying temperature accelerated binder migration to the coating surface, however low drying temperature made binder migrate towards the interior of base paper. Moderate drying temperature is good to more even binder distribution and coating layer forming.
\end{abstract}

\section{Introduction}

Binder is the essential component of coating color recipe. The amount and type of latex binder has a significant effect on both the structure and chemical-physical properties of coated paper, which affect critical surface properties, e.g., print gloss, roughness, ink setting rate via liquid absorption, and print mottle [1-2]. Binder migration towards base paper will contribute to the ink absorption and ink usage increasing ink absorption, and decrease printing gloss. Binder migration towards coating surface can result in printing mottles [3-4]. Some researchers have studied the binder distribution in recent years. Hagen $[5,6]$ has investigated different drying mechanisms and found that drying strategy and especially the drying rate near the gel point play an important role. The binder was found to be significantly affected coating structure and print quality based on AFM phase imaging and TOF-SIMS mapping [7, 8]. In these researches, some treatments must be done before measurements, which might destroy paper structure and do some disadvantages to measurements. In this study, To observe binder distribution, and obtain the relationship between drying condition and binder migration, Rhodamine B was used to stain binder in this study. This method would not need to cut the paper and embed the sample into the resin to obtain the cross-section. The main aim of the investigation was to characterize the z-directional distribution of the binder and obtain the influence of drying condition of coated paper on the binder migration.

\section{Experimental}

Coating Samples. The basis weight of uncoated base paper (supplied by Dong Tang Paper Mill) was $70 \mathrm{~g} / \mathrm{m}^{2}$. The coatings of paper were composed with kaolin pigment (supplied by Mao Ming Clay Company, China) and carboxylic styrene-butadiene latex (supplied by BASF Company, China). The coating components are indicated in Table 1. Rhodamine B (Sigma-R6626, Co. Ltd) was used as fluorescent dye for staining with the binder because it was successfully applied in some studies [9, 10]. Rhodamine B was dissolved in ethanol to reach $0.05 \mathrm{wt} \%$. 0.05wt\% of Rhodamine B was added to carboxylic styrene-butadiene latex, and then it was stirred. The binder was held to stain completely for $24 \mathrm{~h}$ before coating in order to make Rhodamine B and binder become the 
whole. Coating was performed with a bar coater (model K303 Multi-coater, RK Print Coat Instruments Ltd, United Kingdom). The coated paper was moved to a drying oven for 1min.

- Coating speed $=5 \mathrm{~m} / \mathrm{min}$

- Drying temperature $=100-220^{\circ} \mathrm{C}$

- Drying time $=1 \mathrm{~min}$

- Coating thickness $=4 \mu \mathrm{m}$

Table 1 Coating color recipes

\begin{tabular}{cccccc}
\hline Sample & Kaolin $\left(\mathrm{pph}^{*}\right)$ & $\mathrm{SB}\left(\mathrm{pph}^{*}\right)$ & Solids(\%) & Coating thickness $(\mu \mathrm{m})$ & Dry temperature $\left({ }^{\circ} \mathrm{C}\right.$ \\
\hline T1 & 100 & 15 & 60 & 4 & 100 \\
T2 & 100 & 15 & 60 & 4 & 130 \\
T3 & 100 & 15 & 60 & 4 & 160 \\
T4 & 100 & 15 & 60 & 4 & 190 \\
T5 & 100 & 15 & 60 & 4 & 220 \\
\hline \multicolumn{2}{r}{ "Parts per hundred }
\end{tabular}

Laser Scanning Confocal Microscopy. A Laser Scanning Confocal Microscopy (Leica TCS-SP5) was used to obtain the z-directional distribution of stained binder. Immersion liquid was supplied by Leica. An X40 oil-immersion objective lens (HC PLAPO, NA 1.25) was chosen. As the laser beam scanned the ink film, the emitted fluorescent light was detected by a photodetector. A confocal beam splitter filter (DD458/514) was used to separate the fluorescent light. The second PMT received He-Ne 514 laser signal, and the detected wavelength ranged from $520 \mathrm{~nm}$ to $615 \mathrm{~nm}$. The appropriate focus plane and the best image could be obtained by adjusting the z-directional position, PMT gain, and PMT offset, respectively. Three-dimensional information was gathered as the optical depth images obtained from the scanner. Confocal images were acquired with the XYZ scanning mode in the $\mathrm{z}$ direction at intervals of $0.2 \mu \mathrm{m}$. A sample was scanned for a period of about 2 to 10 minutes, depending on the thickness of coating film, Z-step, speed, and pixel format of scanning. The following scanning conditions were selected: pinhole size $=67.95 \mu \mathrm{m}$; digital zoom $=$ 1 ; scan area $=386 \times 386 \mu \mathrm{m}^{2}$; scanning pixel format $=1024 \times 1024$; scanning speed $=400 \mathrm{~Hz}$; laser intensity $=70 \%$; Z-step $=0.2 \mu \mathrm{m}$; and excitation wavelength $=514 \mathrm{~nm}$.

\section{Results and Discussion}

Visual characterization of the Z-directional Distribution evenness of Binder by LSCM. Fig. 1 shows the fluorescent intensity distribution in the z-direction (thickness direction) and illustrates the $z$-directional binder profiles after image processing and analysis using leica software. The binder z-directional distribution profiles in Fig. 1 obtained after the samples were measured with the XYZ scanning mode of LSCM in the z-direction at intervals of $0.2 \mu \mathrm{m}$. Binder was stained with Rhodamine B, so the fluorescent signals detected by LSCM represented the binder exiting. When laser scanned the coating surface, variation of the fluorescent intensity showed the variation of the amount of the binder in the z-direction. Fig. 1 summarizes the effect of drying temperature on the binder content and the binder uniformity in the z-direction as measured by LSCM.

The z-direction binder profiles suggest that there is a correlation between drying temperature and binder distribution. The coating thickness of overall samples was $4 \mu \mathrm{m}$, so the binder must penetrate into the base paper when z-direction length detected by LSCM was larger than $4 \mu \mathrm{m}$. The samples T1 and T2 yielded a higher depth value of binder z-directional penetration into base paper compared with the other samples (see Fig. 1). The z-direction length of sample T1 is the largest, which indicate that it is the highest degree of binder migration to the base paper in coating sample T1. The z-direction length of sample T5 is the lowest, which indicate that it is the lowest degree of binder migration to the base paper in coating sample T5. The correlation was observed from the two aspects that describe uniformity: symmetry of profile changing and smooth degree of the profile for the z-directional binder distribution. More unevenness in the binder distribution can create higher local variations in pore structure, and in turn, spatial variations in absorption that can yield mottling. The unexpected binder migration can be inferred from the profiles (see Fig. 1). The LSCM can be 
applied to analyze the degree of binder migration in coated papers. A coated paper binder profile with variations in the $\mathrm{z}$ - direction variations observed will give an indication of the presence of binder migration.
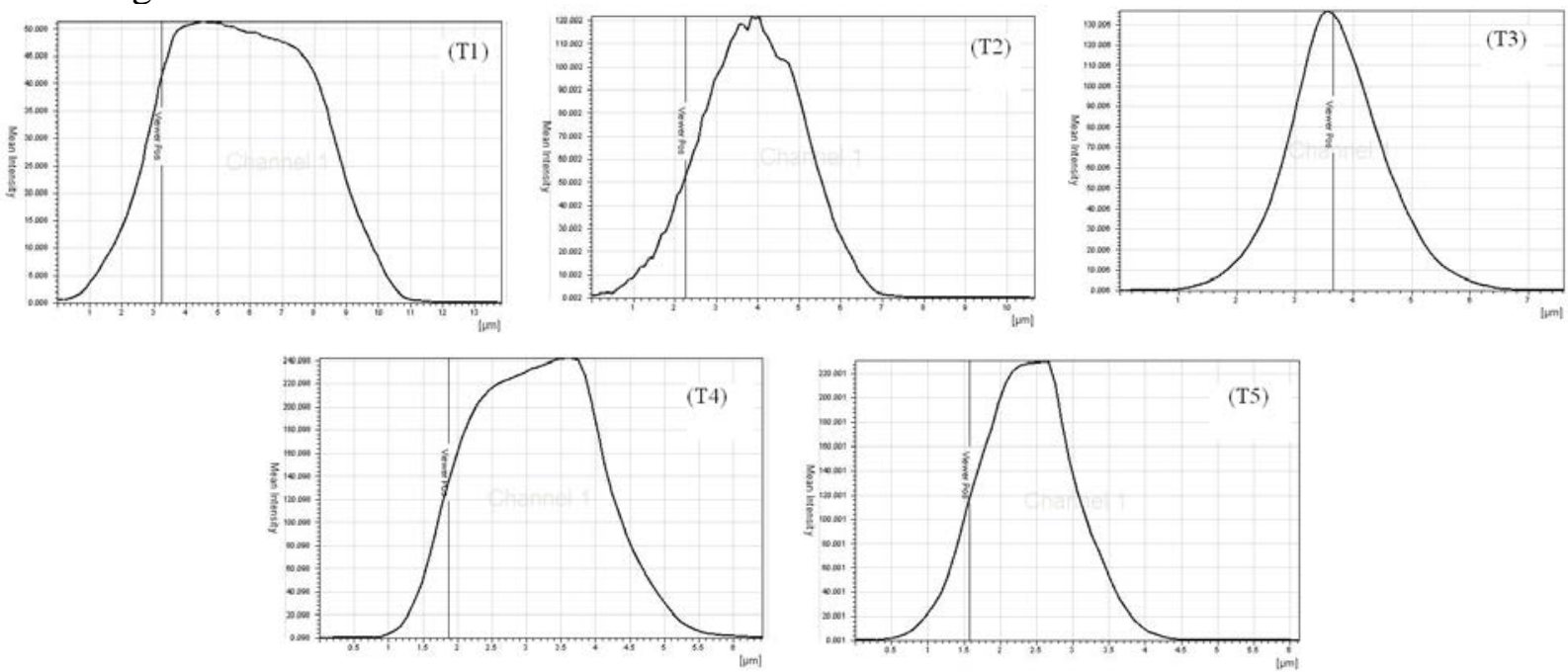

Fig.1. Profiles of the binder z-directional distribution of coating samples which dried at different temperature.

Numerical characterization of the Z-directional Distribution depth of Binder by LSCM. Average penetration depth of binder in the z-direction showed in Table 2 was obtained by the method of measuring thirty points of five samples. Average penetration depth in base paper of sample T5 was $0.02 \mu \mathrm{m}$, so there is no phenomenon of binder migration towards base paper in sample T5. Average penetration depth in base paper of sample T1 was7.27 $\mu \mathrm{m}$, which is the biggest value in the samples. It could be concluded that lowest drying temperature resulted in highest amount of the binder penetration into base paper and more uneven distribution of binder. This result was a consequence of that water had longer time in the coating layer, which made water to carry binder to penetrate into the base paper (absorption). When sample T5 is compared with the others, the findings suggest that high drying temperature accelerates water to carry binder migration to coating surface (evaporation). The resulting balance between the two opposite driving forces, absorption and evaporation, governs the movement of the liquid phase within the coating layer as consolidation proceeds, and in turn, it determines the binder spatial distribution in real coated papers [11,12]. It is also found that sample T3 creates a more uniform binder arrangement in the $z$-direction in the drying condition of $160^{\circ} \mathrm{C}$. The driving forces between absorption and evaporation got balance in the moderate drying condition. The high drying temperature and low drying temperature were propitious to the preferential movement of the binder to the base paper (absorption) and to the top surface of the coating (evaporation), respectively. Mild drying temperature will resulted in moderate binder distribution and more even changes of binder distribution, which would produce better print quality.

Table 2 Average penetration depth of binder in the Z-direction

\begin{tabular}{cccc}
\hline Samples & $\begin{array}{c}\text { Z-direction average } \\
\text { penetration depth }(\mu \mathrm{m})\end{array}$ & $\begin{array}{c}\text { Coating } \\
\text { thickness }(\mu \mathrm{m})\end{array}$ & $\begin{array}{c}\text { Average penetration depth } \\
\text { in base paper }(\mu \mathrm{m})\end{array}$ \\
\hline T1 & 11.27 & 4 & 7.27 \\
T2 & 7.26 & 4 & 3.26 \\
T3 & 5.25 & 4 & 1.25 \\
T4 & 4.12 & 4 & 0.12 \\
T5 & 4.02 & 4 & 0.02 \\
\hline
\end{tabular}

\section{Conclusions}

Laser Scan Confocal Microscopy played an important role in investigation into the z-directional 
distribution depth and evenness of binder in coated paper related to drying temperature in this experiment. The z-directional distribution depth and evenness of binder was showed in the method of visual and numerical characterization by LSCM. A coated paper binder profile with variations in the z- direction variations observed gave an indication of the presence of binder migration. A clear increasing trend of binder content in base paper interior was observed in terms of decreasing the drying temperature. Based on the results presented in this study, low and high drying temperature seemed to be less desirable because they produced more uneven binder distributions that could potentially result in larger printing defects. Mild drying temperature was advantageous for binder even distribution and decrease the degree of binder migration, which would produce good print quality.

\section{References}

[1] J. Watanabe and P. Lepoutre: Journal of Applied Polymer Science 27 (1982) p.4207-4219.

[2] P. Heikkil€a and N. Milosavljevic: Drying Technology 20 (1) (2002) p.211-222.

[3] G. Engstr€om: Tappi Journal 77 (4) (1994) p.160-172.

[4] S. Di Risio and N. Yan: Collids and Surfaces A: 289(2006) p.65-74.

[5] K.G. Hagen: Proceedings of Tappi 1985 Coating Conference, p.131-137.

[6] K.G. Hagen: Tappi 1990 Blade Coating Seminar Notes, Tappi Press, Atlanta, p.125-131.

[7] C. Kugge: J. Pulp Paper Sci. 30 (4) (2004) p.105-111.

[8] R.N.S. Sodhi, L. Sun, M. Sain and R. Farnood: J. Adhesion 84 (3) (2008) p.277-292.

[9] Y. Ozaki, D.W. Bousfield and S.M. Shaler: Journal of pulp and paper science 31(1) (2005) p.48-52.

[10] Y. Ozaki, D.W. Bousfield and S.M. Shaler: TAPPI J. 5(2) (2006) p.3-8.

[11] G. Engstr"om, M. Rigahl, J. Kline and J. Ahlroos: Tappi J. 5 (1991) p.171-179.

[12] M. Whalen-Shaw, TAPPI Press, Atlanta, 1993. 